\title{
Modelo matemático para el control químico con resistencia del Aedes aegypti (Diptera: Culicidae)
}

\section{A mathematical model for the chemical control of Aedes aegypti (Diptera: Culicidae) having acquired chemical resistance}

\author{
Leonardo D. Restrepo-Alape, Hernán D. Toro-Zapata y Aníbal Muñoz-Loaiza
}

Departamento de Matemáticas Facultad de Ciencias Básicas y Tecnologías Universidad del Quindío. Armenia, Colombia. ldrestrepo@uniquindio.edu.co, hdtoro@uniquindio.edu.co, anibalml@hotmail.com

Recibido 10 Mayo 2010/Enviado para Modificación 12 Diciembre 2010/Aceptado 23 Diciembre 2010

\section{RESUMEN}

El dengue es una enfermedad viral común en zonas tropicales y subtropicales transmitida por mosquitos del género Aedes. El virus es transmitido a los humanos por la picadura de un mosquito hembra infectado. Ya que no existen vacunas que protejan contra la infección, el control de la enfermedad se hace controlando la población adulta o inmadura del mosquito. En este trabajo se modela la dinámica de crecimiento del mosquito sometido a control adulticida y con resistencia al químico. Se hace el análisis del modelo mediante análisis clásico de estabilidad local de sistemas dinámicos, lo que permite determinar el umbral de crecimiento del mosquito y a partir de éste establecer una estrategia adecuada de control químico. Se incluye la simulación numérica para diferentes escenarios con el fin de evaluar si hay diferencias en el comportamiento del sistema cuando la resistencia está presente y cuando no lo está.

Palabras Clave: Modelos matemáticos, Dengue, control de vectores (fuente: DeCS, BIREME).

\section{ABSTRACT}

Dengue fever is a common vector-borne disease in tropical and subtropical areas. It is transmitted to humans by the bite of an infected female Aedes mosquito. Since no vaccines are currently available which can protect against infection, disease control relies on controlling the mosquito population. This work was aimed at modelling such mosquito's population dynamics regarding chemical control of the adult population and its acquired resistance to chemicals. The model was analysed by using classical dynamic system theory techniques and mosquito growth threshold was determined as this establishes when a particular population may prosper in the environment or when it is likely to disappear. A suitable chemical control strategy was developed from such threshold. Simulations were made in control and non-control scenarios; this determined the degree of control application effectiveness against different levels of acquired resistance.

Key Words: Mathematical model, dengue, vector control (source: MeSH, NLM). 
$\mathrm{E}$ 1 dengue es actualmente una de las enfermedades que afectan al hombre y constituye un severo problema de salud pública en el mundo, especialmente en zonas tropicales y subtropicales donde las condiciones del medio ambiente favorecen el desarrollo y la proliferación del Aedes aegypti, el cual es el principal mosquito vector. El agente causal es un virus de la familia Flaviviridae: arbovirus (arthropod-borne); se reconocen por variación de la proteína E, 4 tipos antigénicos llamados DEN-1, DEN-2, DEN-3 o DEN-4, los cuales son transmitidos a los humanos por la picadura de un mosquito infectado (1).

El mosquito presenta los siguientes estados de desarrollo: huevos, larvas, pupas, (fase acuática) y finalmente mosquitos maduros (fase aérea). Este proceso lo lleva a cabo en ambientes adecuados para su desarrollo y proliferación de la población $(2,3)$.

Las entidades de salud pública del mundo se han puesto en la tarea de disminuir la incidencia del dengue, para ello recurren a varios tipos de controles, ya sea de manera manual o por la utilización de insecticidas. Mediante el primer método se pueden destruir los criaderos, método que depende de la participación de la población humana, mientras que el uso de los insecticidas ha tenido mayor éxito en controlar el vector. Pero con el paso del tiempo, los vectores adquieren resistencia a estos químicos (de generación en generación), volviéndose más resistentes y constituyendo una limitante importante para su control.

En cuanto al modelado del dengue y el mosquito transmisor se encuentran trabajos sobre: la transmisión vertical en el mosquito, la dinámica de transmisión espacio-temporal, la dinámica de transmisión de los diferentes serotipos del virus, la influencia del clima en la ocurrencia del dengue, la dinámica de estructura poblacional, etc ${ }^{1}$. (4-8). Particularmente, el modelado sobre el control del mosquito se ha hecho utilizando el efecto de adulticidas y larvicidas en las tasas de mortalidad constantes (9-11); mediante control de los criaderos (12) y aplicando el principio del máximo de Pontryagin² (13-15), entre otras.

\footnotetext{
${ }^{1}$ Ruiz J. Modelo estocástico de transmisión del dengue en poblaciones estructuradas. Tesis Doctoral, Doctorado en Ciencias, área: Biotecnología. Universidad de Colima. Biblioteca Central. Tecomán, Colima, México. 2004.

${ }^{2}$ Muñoz A. Modelado matemático del dengue clásico. Tesis doctoral, Doctorado en Ciencias Matemáticas, Facultad de Ciencias Físico-Matemáticas (FCFM). Benemérita Universidad Autónoma de Puebla (BUAP). Biblioteca de la FCFM-BUAP. Puebla, México, 2007.
} 


\section{El modelo}

El modelo considera una población de mosquitos dividida en dos estados, uno correspondiente a los mosquitos adultos en cualquier tiempo $t$, denotado con $x(t)$ y otro correspondiente a los mosquitos inmaduros (huevos, larvas y pupas) en cualquier tiempo $t$, denotado con $y(t)$. Se aplica control químico sobre la población adulta del mosquito (control adulticida).

El crecimiento de la población de mosquitos adultos está descrito por el término $f \omega y$, donde $\omega y$ describe la proporción de mosquitos que alcanzan la madurez y $f$ es la fracción de éstos mosquitos que no son resistentes al control químico aplicado; de manera que el mosquito adulto muere por causas ajenas al control a una tasa e y por efecto del control $u$. De este modo la ecuación diferencial que describe la variación en el tiempo de la población de mosquitos adultos es,

$$
\frac{d \mathrm{x}}{d t}=\mathrm{f} \omega \mathrm{y}-\varepsilon \mathrm{x}-\mathrm{ux}
$$

La variación de la población de mosquitos inmaduros en el tiempo se desarrolla siguiendo una ley logística con una tasa de crecimiento $\varphi=\varphi(t)$ y con capacidad de carga $k$, la población de mosquitos inmaduros mueren a una tasa natural $\beta$, y madura a una tasa $\omega$, de este modo el término $\beta$ y representa el número promedio de individuos que mueren por causas naturales y el término wy es el número promedio de individuos que pasa al estado adulto. La ecuación diferencial que describe la población de mosquitos inmaduros es,

$$
\frac{d \mathrm{y}}{d t}=g \varphi \times\left(1-\frac{y}{k}\right)-(\beta+\omega) \mathrm{y}
$$

donde $g$ es la fracción de mosquitos inmaduros que no tienen resistencia al químico. El sistema completo es:

$$
\left\{\begin{array}{l}
\dot{x}-f \omega y-(\varepsilon+u) x \\
\dot{y}-g \varphi x-\left(1-\frac{y}{k}\right)-(\beta+\omega) y
\end{array}\right.
$$

$\operatorname{con} f, \varepsilon, \omega, g, \phi, \beta, \mathrm{k}>0 ; f, g, \mathrm{u} \in[0,1]$. 
Umbral de crecimiento del mosquito

Para $\varphi$ constante y el control $u=0$, encontramos las soluciones estacionarias haciendo $\frac{d \mathrm{x}}{d t}=0$ y $\frac{d \mathrm{y}}{d t}=0$ en el sistema [1], con lo que se tiene que el

equilibrio trivial es,

$$
\left(\mathrm{x}^{0}, \mathrm{y}^{0}\right)=(0,0)
$$

Este equilibrio corresponde a la ausencia de poblaciones en el medio. Por otro lado, el equilibrio no trivial está dado por,

$$
\left(x^{1}, y^{1}\right)=\left(\frac{f \omega k}{(\varepsilon+u) \sigma}(\sigma-1), \frac{k}{\sigma}(\sigma-1)\right)
$$

donde $\sigma=\frac{\operatorname{gf} \omega \varphi}{(\varepsilon+u)(\beta+\omega)}$ y corresponde al equilibrio en presencia de las poblaciones. La cantidad $\sigma$ es el umbral de crecimiento del mosquito; en efecto, si $\sigma \leq 1$, el único punto de equilibrio con sentido biológico es el trivial, lo que implica la extinción de la especie. La solución de coexistencia tiene sentido biológico si $\sigma>1$.

El análisis de estabilidad local de los equilibrios conduce al siguiente resultado:

Teorema 1. El equilibrio trivial del sistema [1] es local asintóticamente estable cuando $\sigma<1$. Cuando $\sigma>1$, el equilibrio trivial es inestable y el equilibrio no trivial es local asintóticamente estable, cuando $\sigma=1$, el equilibrio trivial es no hiperbólico.

Demostración. La matriz jacobiana del sistema [1] en el equilibrio trivial es:

$$
J_{0}\left(x^{0}, y^{0}\right)=\left(\frac{-(\varepsilon+u)}{g \varphi} \frac{f \omega}{-(\beta+\omega)}\right)
$$

Utilizando el criterio traza-determinante se tiene que $\operatorname{Tr}\left(\mathrm{J}_{0}\right)=-(\varepsilon+\mathrm{u}+\beta+\omega)<0$ $y \operatorname{det}\left(J_{0}\right)=(\varepsilon+u)(\beta+\omega)(1-\sigma)$ por lo tanto el determinante es mayor que cero si y lo que lo hace ser asintóticamente estable. Si $\sigma>1$ es inestable. Cuando $\sigma=1$ el determinante es cero por lo tanto el equilibrio trivial es no hiperbólico. 
Para la solución no trivial se utiliza nuevamente el criterio traza-determinante. La matriz jacobiana es:

$$
J_{1}\left(x^{1}, y^{1}\right)=\left(\begin{array}{cc}
-(\varepsilon+u) & f \omega \\
\frac{g \varphi}{\sigma} & -\sigma(\beta+\omega)
\end{array}\right)
$$

De donde se tiene que la traza está dada por $\operatorname{Tr}\left(\mathrm{J}_{1}\right)=-(\varepsilon+\mathrm{u}+\sigma(\beta+\omega))$, y el determinante por $\operatorname{det}\left(\mathrm{J}_{1}\right)=(\varepsilon+u)(\beta+\omega)(\sigma-1)$, por lo tanto el determinante es mayor que cero si $\sigma>1$ con lo que el equilibrio no trivial es asintóticamente estable. Si $\sigma<1$ es inestable y cuando $\sigma=1$ el equilibrio no trivial colapsa en el origen con el equilibrio trivial; en este caso el determinante es cero por lo tanto es no hiperbólico.

Debe entenderse entonces como una estrategia efectiva de control aquella que garantice que $\sigma<1$, lo que garantiza el control de la población de mosquitos. Para establecer esta estrategia basta determinar $u$ de tal manera que $\sigma<1$. Despejando u de

$$
\frac{\operatorname{gf} \omega \varphi}{(\varepsilon+u)(\pi+\omega)}<1
$$

se obtiene,

$$
\mathrm{u}<\frac{\mathrm{gf} \omega \varphi}{(\pi+\omega)}-\varepsilon
$$

Este resultado, llamado criterio de control químico se resume en la siguiente proposición.

Proposición. Dado el sistema [1], el control u que garantiza que $x(t)$ y y(t) tiendan a cero cuando $t$ crece está dado por

$$
\text { a }>\frac{g f \omega \varphi}{(\pi+\omega)}-\varepsilon
$$

La expresión [2] permite determinar el valor adecuado u para controlar la población de mosquitos no resistentes, siempre que se conozcan los valores de los parámetros y las fracciones de mosquitos adultos e inmaduros que no adquieren resistencia al químico. 
Simulación numérica sin control

La simulación del modelo se hizo utilizando el programa MATLAB. Los valores de los parámetros se muestran en la Tabla 1 y corresponden a valores extraídos de la literatura (13).

Tabla 1. Significado y valores asignados a las variables y a los parámetros

\begin{tabular}{llc}
\hline \multicolumn{1}{c}{ Variables y parámetros } & Valor \\
\hline$x$ & Número promedio de mosquitos adultos en un tiempot & 300 \\
$y$ & Número promedio de mosquitos inmaduros en un tiempot & 300 \\
$f$ & Fracción de mosquitos inmaduros que no desarrollan resistencia & 1 y 0.7 \\
$g$ & Fracción de huevos sin resistencia al químico & 1 y 0.7 \\
$\omega$ & Tasa de desarrollo de los estados inmaduros. & 0.4 \\
$\varepsilon$ & Tasa de mortalidad de los mosquitos maduros. & 0.1 \\
$k$ & Capacidad de carga de los mosquitos inmaduros. & 1000 \\
$\pi$ & Tasa de mortalidad de los mosquitos inmaduros. & 0.1 \\
$\varphi$ & Ovoposición de los mosquitos maduros sin y con resistencia & 0.9 \\
$u$ & Control químico & $0 ; 0,56$ y 0,2 \\
\hline
\end{tabular}

Combinaciones

El análisis numérico se hizo teniendo en cuenta el comportamiento de las poblaciones $\mathrm{X}(t)$ y $\mathrm{y}(t)$ en un periodo de dos años y siguiendo una tasa de ovoposición de los mosquitos maduros constante y con los parámetros usados por Caetano (13).

La Figura 1 muestra que cuando no se aplica control, la población de mosquitos crece hasta niveles altos. En las gráficas a y b se muestra el comportamiento de las dos poblaciones si se considera que la tasa de crecimiento $\varphi$ es constante. Por otro lado, en las gráficas c y d se muestra el comportamiento de las poblaciones cuando se asume una función periódica,

$\varphi(t)=\rho(1-\delta \sin (\alpha t-\theta))$

con $\delta=0,08, \alpha=\frac{2 \pi}{52}, \rho=0,9$ y $\theta=0$. Esta función fue propuesta por Caetano en (13) para describir el crecimiento de la población de mosquitos, considerando la influencia de los cambios estacionales.

Es importante ver que las oscilaciones que se observan en las graficas c y d de la Figura 1, representan la respuesta de las poblaciones a la estacionalidad considerada en la función [3]; sin embargo, el comportamiento en ambos casos es cualitativamente equivalente. 
Figura 1. Gráfica de las funciones $x(t)$ y $y(t)$ sin incluir control ni resistencia. Las gráficas a y b consideran $\varphi$ constante y las gráficas $c$ y d consideran la función [2]
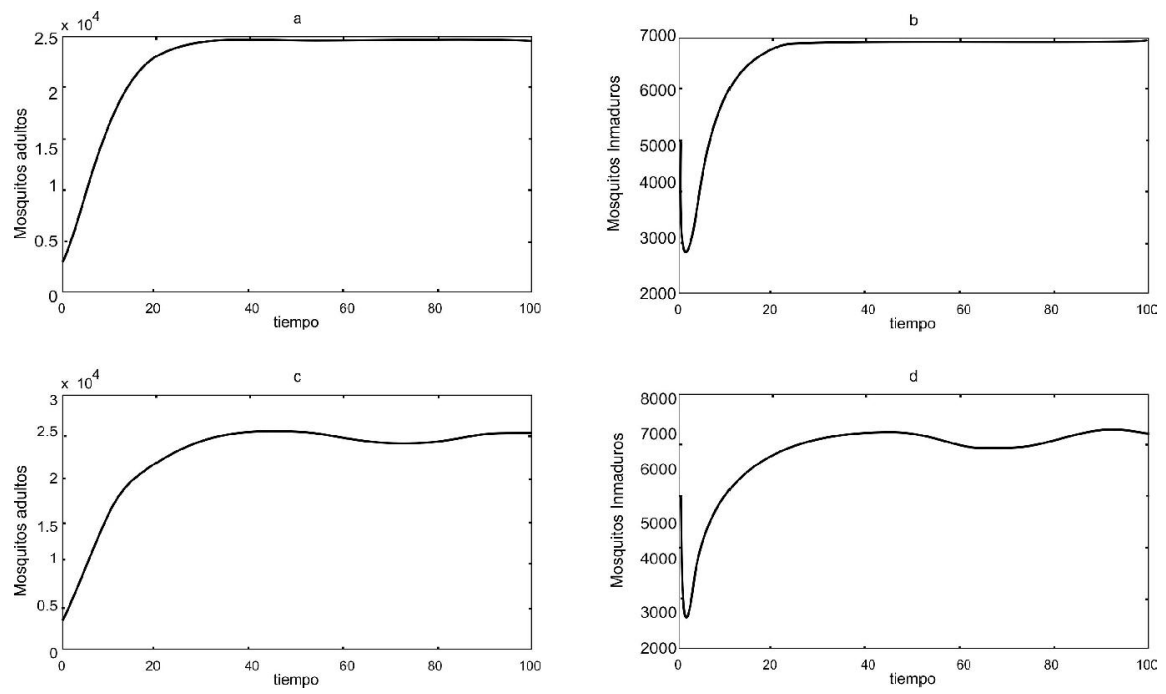

Simulación con control

Si se considera que los mosquitos no adquieren resistencia al químico, es decir que $f=1$ y $g=1$, se tiene por la expresión [2] que $\square \geq 0.56$. Con esta consideración se hace la simulación de la Figura 2, en la cual se observa como efectivamente, el control aplicado es efectivo en la reducción de la población de mosquitos.

Considerar que las poblaciones adquieren cierto grado de resistencia, de manera que $f=0.7$ y $g=0.7$, implica que la población de mosquitos no resistentes es menor. En este caso $u \geq 0.1469$, da resultados muy semejantes a los que se muestran en la Figura 2. Sin embargo se aplicara un control un poco más alto, por ejemplo $\square \geq 0.2$ se tendría que la población de mosquitos es controlada más rápidamente, como puede verse en la Figura 3.

Es importante observar que el aumento de adquisición de resistencia (reducción en los valores de $f$ y $g$ ) implica que las poblaciones no resistentes son más fáciles de controlar y por tal motivo, se reduce el valor de requerido para este fin. 


\section{Conclusiones}

Mientras no haya control químico las poblaciones de mosquitos tendrán un crecimiento alto y posiblemente dará paso a epidemias, ya que la enfermedad es transmitida por el mosquito maduro, es recomendable utilizar estrategias de control químico para reducir el crecimiento de los mosquitos y de manera indirecta los costos del tratamiento de la enfermedad.

Figura 2. Gráfica de las funciones $x(t)$ y $y(t)$ y con control pero sin resistencia. Las graficas a y b consideran $\varphi$ constante y las gráficas $c$ y d consideran la función [2]. El valor del control es establecido con la condición [3]
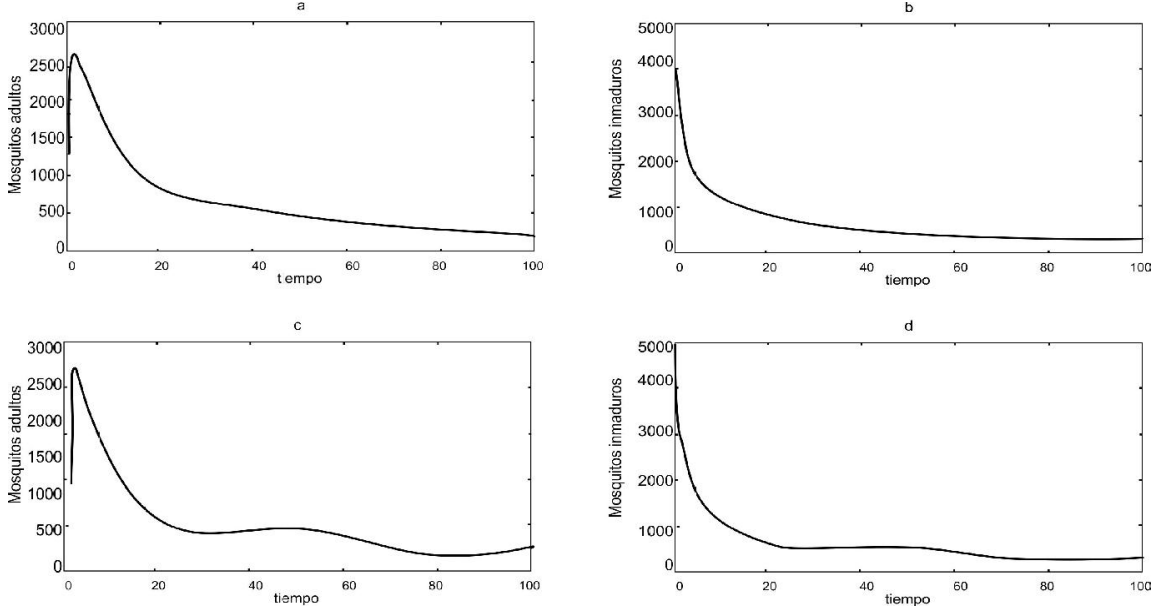

Figura 3. Funciones $x(t)$ y $y(t)$ y con control y con resistencia. Las graficas a y b consideran $\varphi$ constante y las gráficas $c$ y d consideran la función [2] El valor del control utilizado es superior al que impone la condición [3]
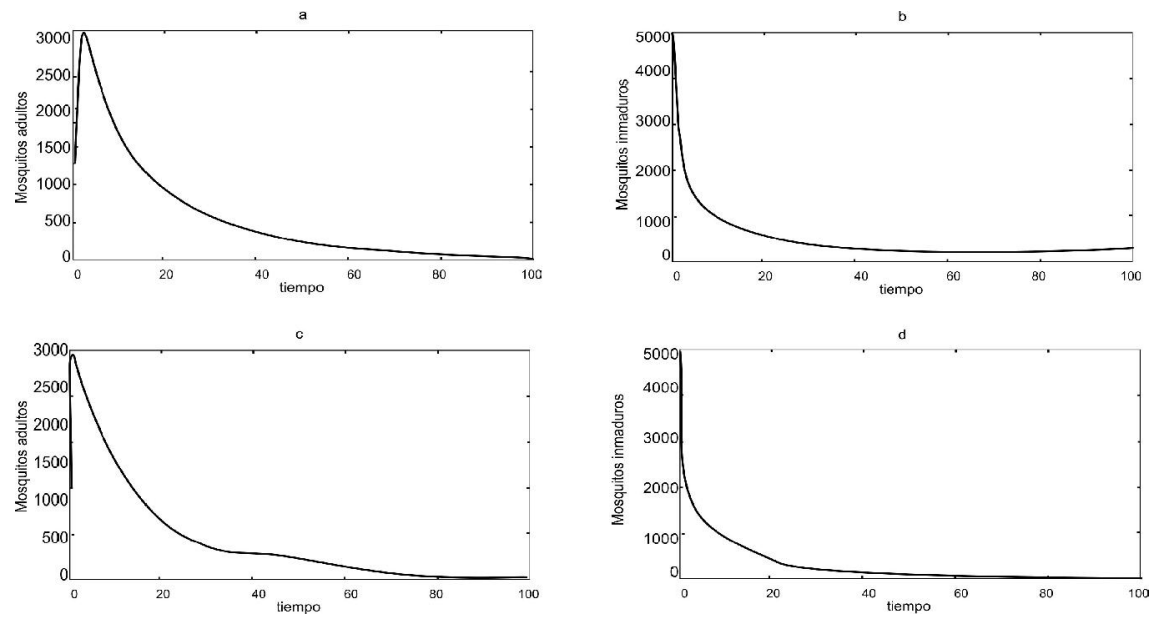
Es claro que el umbral de crecimiento está sujeto a los parámetros y variables del modelo, por lo que es importante contar con una clara determinación de estos parámetros, con el fin que el modelo sea aplicable en situaciones específicas.

Es sabido que aumentar la aplicación de control químico, aumenta la resistencia del mosquito, y como se ha visto esto hace que sea más fácil reducir las poblaciones no resistentes, es decir, se hace necesaria una menor inversión en químicos. Este hecho no es nada alentador, porque implica que las poblaciones de mosquitos se están haciendo resistentes y no es posible controlarlas por este mecanismo. En resumen, el control químico, tan efectivo como parece, pierde paulatinamente su efectividad y hace que sea necesario encontrar otros mecanismos de control que no dependan de la resistencia al químico que tenga el mosquito *

\section{REFERENCIAS}

1. Huntingford C, Hemming D, Gash JHC, Gedney N, Nuttall PA. Impact of climate change on health: what is required of climate modellers? Transactions of the Royal Society of Tropical Medicine and Hygiene. 2007; 101(2):97-103.

2. Takahashi LT, Maidana NA, Ferreira Jr, WC. O Aedes e sua onda. Biomatemática: 2003; XIII: 3344.

3. Chowel G. Estimation of the reproduction number of dengue fever from spatial epidemic data. Mathematical Biosciences. 2007; 208:571-589.

4. Pongsumpan IM, Tang P. Transmission of dengue hemorrhagic fever in an age structured population. Journal of Mathematical and Computer Modelling. 2003; 37(9-10):949-961.

5. Esteva L, Vargas C. Influence of vertical and mechanical transmission on the dynamics of dengue disease. Mathematical Biosciences. 2000; 167:51-64.

6. Wu P.C. Weather as an effective predictor for occurrence of dengue fever in Taiwan. Acta Trópica. 2007; 103:50-57.

7. Esteva L, Vargas C. Coexistence of different serotypes of dengue virus. J. Math. Biol. 2003; 46:31-47.

8. Feng Z, Velasco-Hernández JX. Competitive exclusion in a vector-host model for the dengue fever. J. Math. Biol. 1997; 35:523-544.

9. Buratani M. Modelling the control strategies against dengue in Singapore. Epidemiol. Infect. 2008; 136:309-319.

10. Yang H. M. Dinamica da transmissao da dengue e delineamento de mecanismos de controle e prevencao. Campinas, Brasil. Ed. UNICAMP/IMECC - DMA. pp. 63-89; 2006

11. Newton EA, Reiter PA. A model of the transmission of dengue fever with an evaluation of the impact of Ultra-Low Volume (ULV) insecticide applications on dengue epidemics. American Journal Of Tropical Medicine and Hygiene. 1992; 47:709-720.

12. Predescu M, Sirbu G, Levins R, Awerbuch-Friedlander T. On the dynamics of a deterministic and stochastic model for mosquito control. Applied Mathematics Letters 2007; 20:919925.

13. Caetano MA, Yoneyama T. Optimal and sub-optimal control in Dengue epidemics. Optimal control applications and methods. 2001; 22:63-73.

14. Gersovitz M, Hammer JS. The economical control of infectious diseases. The Economic Journal. 2004; 144:1-27.

15. Greenhalgh D. Some results on optimal control applied to epidemics. Mathematical Biosciences. 1986; 88:125-158. 